\title{
ChemComm
}

RSCPublishing

COMMUNICATION

View Article Online

View Journal | View Issue

Cite this: Chem. Commun., 2013, 49, 6436

Received 14th April 2013, Accepted 24th May 2013

DOI: $10.1039 / c 3 c c 42732 c$

\section{The first purification and unequivocal characterization of the radical form of the carbon-centered quinone ketoxy radical adduct $\dagger$}

\author{
Chun-Hua Huang, ${ }^{a}$ Guo-Qiang Shan, ${ }^{a}$ Li Mao, ${ }^{a}$ Balaraman Kalyanaraman, ${ }^{b}$ \\ Hao Qin, ${ }^{a}$ Fu-Rong Ren ${ }^{a}$ and Ben-Zhan Zhu*ac
}

www.rsc.org/chemcomm

We found, unexpectedly, that the radical form of the carboncentered quinone ketoxy radical adduct with a recently developed spin-trapping agent BMPO can not only be directly detected and identified using HPLC/high resolution MS, but can also be isolated and purified using semi-preparative HPLC, enabling direct observation of its clean 6-line ESR signal.

Halogenated quinones are a class of toxicological intermediates which can cause acute hepatoxicity, nephrotoxicity, and carcinogenesis. ${ }^{1,2}$ They have also been observed as reactive oxidation intermediates or products in processes used to oxidize or destroy polychlorinated persistent organic pollutants in various chemical and enzymatic systems. ${ }^{1-4}$ More recently, several polyhalogenated quinones, which are suspected bladder carcinogens, were identified as new chlorination disinfection byproducts in drinking water. ${ }^{4}$

We have recently shown that halogenated quinones could enhance the decomposition of hydroperoxides and formation of alkoxyl/hydroxyl radicals independent of transition metal ions. ${ }^{5-8}$ A new carbon-centered quinone ketoxy radical $\left({ }^{\bullet} \mathrm{CBQ}\right.$ $\mathrm{OH}, \mathrm{MW}: 157)$ adduct with the spin-trapping agent DMPO (5,5-dimethyl-1-pyrroline $\mathrm{N}$-oxide) (simply referred to here as DMPO-157) was observed during the reaction between 2,5-dichloro-1,4-benzoquinone (DCBQ) and $t$-butylhydroperoxide $(t$-BuOOH $){ }^{7}$ However, although we can observe the ESR signal for DMPO-157, we cannot directly detect its nitroxide radical form, but only its corresponding more stable non-radical nitrone form, by mass spectrometry (MS). Similarly, we can isolate only the ESR-silent DMPO-157 nitrone adduct by HPLC, but not its corresponding ESR-active nitroxide form. ${ }^{7}$ In other words, we could not obtain the pure DMPO-157 nitroxide radical adduct so that we can observe its clean 6-line ESR signal without any interference of other concurrently formed radicals such as

\footnotetext{
${ }^{a}$ State Key Lab of Environmental Chemistry and Ecotoxicology, Research Center for Eco-Environmental Science, CAS, Beijing 100085, China. E-mail: bzhu@rcees.ac.cn; Fax: +86-10-62923563; Tel: +86-10-62849030

${ }^{b}$ Dept of Biophysics, Medical College of Wisconsin, Milwaukee, WI 53226, USA

${ }^{c}$ Linus Pauling Inst, Oregon State Univ, Corvallis, OR 97331, USA

$\dagger$ Electronic supplementary information (ESI) available. See DOI: 10.1039/ c3cc $42732 \mathrm{c}$
}

$t$-butoxyl and methyl radicals. The reason might be that either the DMPO-157 nitroxide radical adduct was not stable enough to pass through the HPLC column and decayed to its ESR-silent nitrone form during the separation process or the concentration of the nitroxide radical adduct in the collected fraction was just too low to be detected by ESR.

It should be more convincing if we can isolate and purify this unusual quinone ketoxy radical with a suitable spin-trapping agent and observe its clean ESR signal without any interference of other concurrently formed radicals, because this will provide more direct and stronger experimental evidence for the existence of such a new kind of quinone radical, which will further support our previously proposed molecular mechanism for metal-independent decomposition of hydroperoxides by halogenated quinones (see Scheme 2 in ref. 7). Therefore, in this study, we plan to address the following questions: can we find a spin-trapping agent which can form a much more stable nitroxide radical adduct with the quinone ketoxy radical? if so, can we directly detect the radical form by MS? and can we isolate and purify it by semi-preparative HPLC, then observe its clean ESR signal directly?

It has been recently shown that the half-lives of several radical adducts with BMPO (5-t-butoxycarbonyl 5-methyl-1-pyrroline $\mathrm{N}$-oxide), a recently-developed spin-trapping agent with a similar chemical structure to DMPO, are much longer than those of their corresponding DMPO adducts. ${ }^{9}$ Therefore, BMPO was chosen to test whether it can form a more stable nitroxide radical adduct with the quinone ketoxy radical ${ }^{\bullet} \mathrm{CBQ}-\mathrm{OH}$.

We found that three radical adducts could be formed by DCBQ and $t$-BuOOH with BMPO, which are similar to that of DMPO (Fig. 1C and E). In comparison with the ESR spectrum of $\mathrm{Fe}(\mathrm{II})-t$-BuOOH-BMPO, which can produce only two radical adducts, BMPO- ${ }^{\bullet} \mathrm{CH}_{3}$ and $\mathrm{BMPO}-t$-BuO ${ }^{\bullet}$, the smaller peaks should be the $\mathrm{BMPO}$ adducts with the quinone ketoxy radical, BMPO- ${ }^{\bullet} \mathrm{CBQ}-\mathrm{OH}$ (Fig. 1C and D). Beyond our expectation, we found that the half-life of the $\mathrm{BMPO}-{ }^{\bullet} \mathrm{CBQ}-\mathrm{OH}$ nitroxide radical adduct is about 5 hours, which is much longer than that for $\mathrm{DMPO}-{ }^{\bullet} \mathrm{CBQ}-\mathrm{OH}$ (half-life: about $15 \mathrm{~min}$ ). It is likely that the electron-withdrawing ability of the butoxycarbonyl group at the carbon-5 position is responsible for the increased stability of the BMPO- ${ }^{\circ} \mathrm{CBQ}-\mathrm{OH}$ adduct. ${ }^{9}$ 


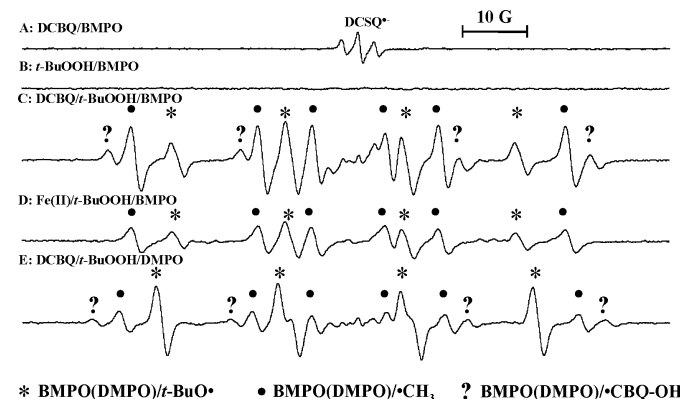

Fig. 1 ESR spectra of ${ }^{\bullet} \mathrm{CBQ}-\mathrm{OH}, t-\mathrm{BuO} \cdot$ and ${ }^{\bullet} \mathrm{CH}_{3}$ radical adducts with BMPO (or DMPO) produced by $\mathrm{DCBQ}$ (or Fe(II)) and $t-\mathrm{BuOOH}$ with spin-trapping agents $\mathrm{BMPO}$ (or DMPO). (A) DCBQ-BMPO; (B) $t-\mathrm{BuOOH}-\mathrm{BMPO}$; (C) DCBQ- $t-\mathrm{BuOOH}-$ BMPO; (D) Fe(II)-t-BuOOH-BMPO; (E) DCBQ-t-BuOOH-DMPO. Reactions were carried out at room temperature in Chelex-treated phosphate buffer $(100 \mathrm{mM}$, pH 7.4). DCBQ, $1 \mathrm{mM}$; Fe(॥), $1 \mathrm{mM}$; $t$-BuOOH, $100 \mathrm{mM}$; BMPO, $50 \mathrm{mM}$; DMPO, $100 \mathrm{mM}$, and $1 \% \mathrm{CH}_{3} \mathrm{CN}$. ESR spectra were recorded $1 \mathrm{~min}$ after the interactions at room temperature under normal room-lighting conditions. The central signal in the spectrum for DCBQ alone was identified as the 2,5-dichlorosemiquinone anion radical $\left(\mathrm{DCSQ}^{\bullet-}\right)\left(a^{\mathrm{H}}=2.01 \mathrm{G} ; g=2.0050\right)$. Hyperfine splitting constants for BMPO- $t$-BuO*: $a^{\mathrm{H}}=14.02, a^{\mathrm{N}}=14.09 ; \mathrm{BMPO}-\mathrm{CH}_{3}: a^{\mathrm{H}}=22.24, a^{\mathrm{N}}=15.61$; BMPO- $\cdot C B Q-O H: a^{\mathrm{H}}=26.5, a^{\mathrm{N}}=16.28 ; \mathrm{DMPO}-t-\mathrm{BuO} \cdot: a^{\mathrm{H}}=16.32, a^{\mathrm{N}}=14.96$; DMPO- $\mathrm{CH}_{3}: a^{\mathrm{H}}=23.56, a^{\mathrm{N}}=16.42 ; \mathrm{DMPO}-\mathrm{CBQ}^{-\mathrm{OH}}: a^{\mathrm{H}}=28.81, a^{\mathrm{N}}=17.11$.

It is well known that spin trapping nitrone-derived adducts could exist in 3 possible redox forms. ${ }^{10}$ Among the 3 redox forms, only the nitroxide form can be detected by ESR, and only the nitrone form was considered as a stable product. Along the same line, the $\mathrm{BMPO}-{ }^{-} \mathrm{CBQ}-\mathrm{OH}$ adduct may exist in 2 of the 3 possible redox forms: the ESR active $\mathrm{BMPO}-{ }^{\circ} \mathrm{CBQ}-\mathrm{OH}$ nitroxide radical form and its corresponding ESR-silent but more stable nitrone form as shown in Scheme 1.

The formation of BMPO-quinone ketoxy radical derived nitroxide and nitrone adducts (simply referred to here as BMPO-157) by DCBQ- $t$-BuOOH-BMPO was first investigated using ESI-Q-TOF-MS. In our previous study with DMPO, we could only detect the ESR-silent nitrone form of DMPO-CBQ-OH (DMPO-157) by MS, but not its corresponding ESR-active nitroxide form. In this study, one-Cl isotope clusters at $\mathrm{m} / \mathrm{z}$ 354 (ESI-negative), which is the nitrone form of BMPO-157, were observed using MS as expected (Fig. 2A). Unexpectedly, a new peak with one-Cl isotope clusters at $\mathrm{m} / \mathrm{z} 355$ was also observed at the same time (Fig. 2A). Based on theoretical calculations, we speculate that the peak at $\mathrm{m} / \mathrm{z} 355$ might be the nitroxide radical form of BMPO-157. It should be noted that

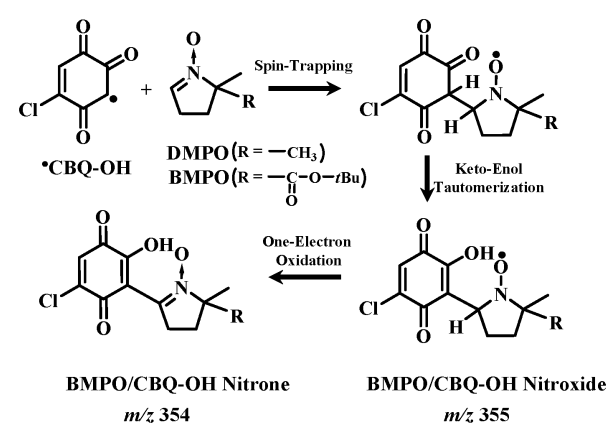

Scheme 1 Proposed pathway for the formation of BMPO nitroxide and nitrone adducts with the carbon-centered quinone ketoxy radical.

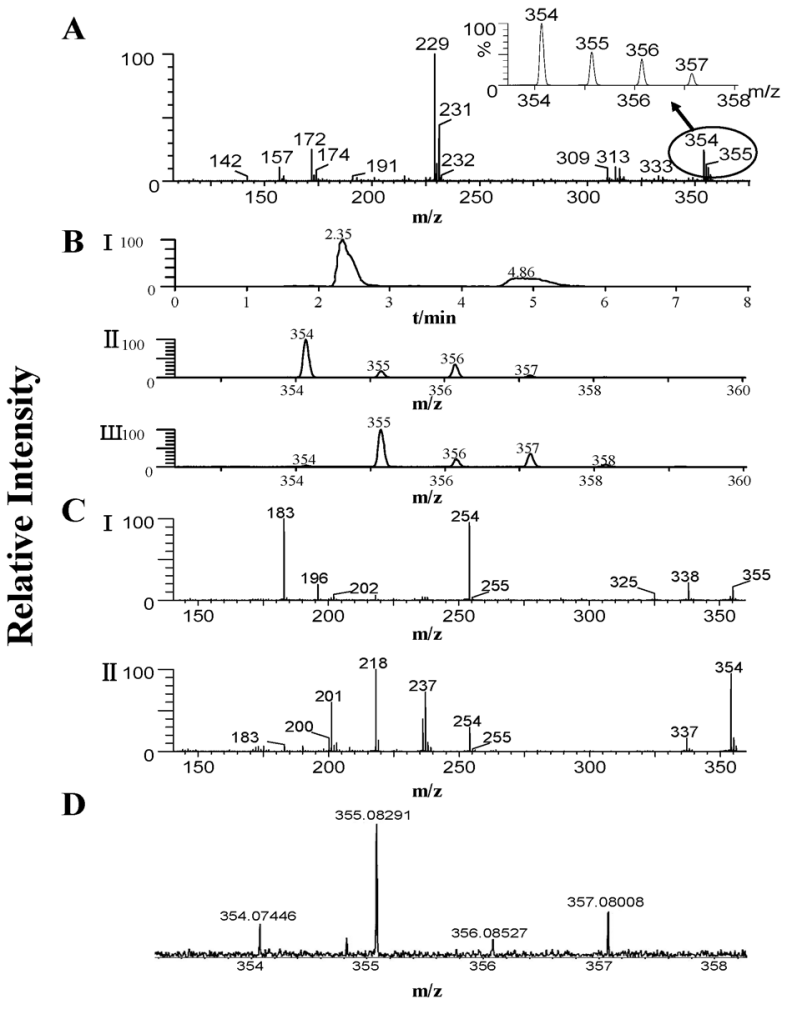

Fig. 2 Identification of two forms of the BMPO-157 adduct by HPLC-ESI-Q-TOFMS and FTICR/MS analyses. (A) The ESI-Q-TOF-MS spectra of BMPO-t-BuOOHDCBQ: BMPO, $50 \mathrm{mM}$; DCBQ, $1 \mathrm{mM}$; $t$-BuOOH, $730 \mathrm{mM}$. (B) (I) Extracted ion chromatography (EIC) $\mathrm{m} / \mathrm{z} 355$ from the reaction of DCBQ (1 mM) with $t-\mathrm{BuOOH}$ $(100 \mathrm{mM})$ in $\mathrm{CH}_{3} \mathrm{COONH}_{4}$ buffer $(\mathrm{pH} 7.4,0.1 \mathrm{M})$ in the presence of $50 \mathrm{mM} \mathrm{BMPO}$. The reaction solution $(30 \mu \mathrm{L})$ was injected into HPLC and eluted with deionized water-acetonitrile (70:30). The ESI-(-)-Q-TOF-MS spectra for the fraction at $2.35 \mathrm{~min}$ (II) and at $4.86 \mathrm{~min}$ (III). (C) The ESI-(-)-Q-TOF-MS/MS spectra of $\mathrm{m} / \mathrm{z}$ at 355 (I) and $m / z$ at 354 (II). (D) The FTICR/MS spectrum of BMPO- ${ }^{\circ B Q-O H}$. Reactions were carried out at room temperature in Chelex-treated phosphate buffer (100 mM, pH 7.4): BMPO, 50 mM; DCBQ, 1 mM; $t-B u O O H, 100$ mM. The fraction was collected by the semi-preparative HPLC method.

the MS peaks correspond to deprotonated, anionic forms of nitrone and nitroxide.

To test whether this is the case, we tried to use HPLC to see whether we can separate the two different forms of BMPO-157. Since the nitroxide radical form of BMPO-157 is susceptible to acid decomposition, a neutral, rather than the traditional acidic, mobile phase was especially chosen for this purpose. Two new peaks with a retention time of 2.35 and $4.86 \mathrm{~min}$, respectively, were observed (Fig. 2B-I). The fraction at $4.86 \mathrm{~min}$ was identified as the nitrone form of BMPO-157, which was characterized using ESI-MS with one-Cl isotope clusters at $\mathrm{m} / \mathrm{z}$ 354 (Fig. 2B-II), while the fraction at $2.35 \mathrm{~min}$ was identified as its corresponding nitroxide radical form, characterized by one$\mathrm{Cl}$ isotope clusters at $m / z 355$ (Fig. 2B-III). The MS/MS studies for $\mathrm{m} / \mathrm{z}$ at 354 and 355 further confirmed that they are two different forms of BMPO-157 (Fig. 2C). These results clearly showed that the two forms of BMPO-157 could be readily separated and identified using HPLC-MS, and the peak with $\mathrm{m} / \mathrm{z}$ at 355 should be the nitroxide radical form of BMPO-157.

Fourier transform ion cyclotron resonance (FTICR) mass spectrometry is one of the techniques that can provide high 


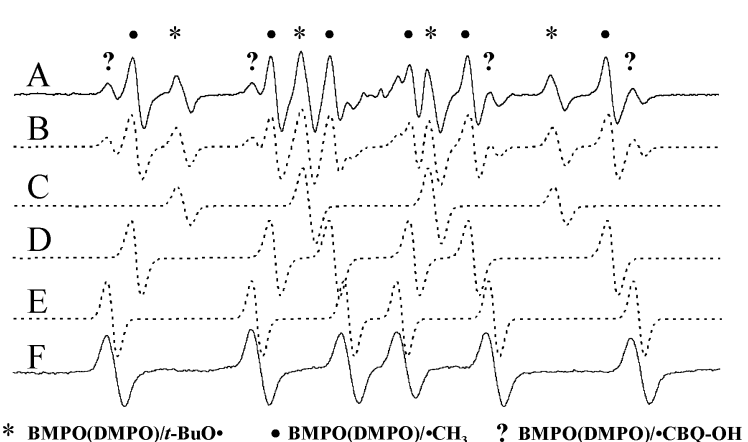

Fig. 3 ESR spectra of BMPO- ${ }^{\circ} \mathrm{CBQ}-\mathrm{OH}$. The ESR spectrum of BMPO-t-BuOOHDCBQ (A). Reactions were carried out in Chelex-treated phosphate buffer (100 mM, pH 7.4). ESR spectra were recorded 1 min after the interactions between $t-\mathrm{BuOOH}$ and $\mathrm{DCBQ}$ at room temperature under normal room-lighting conditions. Simulation of the ESR spectrum of BMPO- $t$-BuOOH-DCBQ (B); BMPO$t$ - $\mathrm{BuO} \bullet(\mathrm{C}) ; \mathrm{BMPO}-\mathrm{CH}_{3}(\mathrm{D})$; BMPO- $\bullet \mathrm{CBQ}-\mathrm{OH}(\mathrm{E})$. The ESR spectrum of the clean $\mathrm{BMPO}-\mathrm{CBQ}^{\mathrm{O}} \mathrm{OH}$ adduct separated from BMPO- $t$-BuOOH-DCBQ (F). The fraction with a retention time of $2.62 \mathrm{~min}(50 \mu \mathrm{L})$ was recorded in ESR after separation by the semi-preparative HPLC method.

mass accuracy and high mass resolution. ${ }^{7,11}$ The BMPO-157 adduct with a retention time of $2.35 \mathrm{~min}$ was characterized using FTICR/MS with one-Cl isotope clusters at $\mathrm{m} / z 355.08291$ (Fig. 2D), which corresponds to the deprotonated molecule of the BMPO-157 nitroxide radical adduct (theoretical value: 355.08281). Interestingly, calculations using Masslynx software automatically suggest that this BMPO-157 adduct is an ion with an odd-electron, further confirming that it is the radical form of the BMPO-157 adduct.

However, we are still not absolutely sure whether the peak at $\mathrm{m} / \mathrm{z} 355$ corresponds to the radical adduct BMPO- ${ }^{\bullet} \mathrm{CBQ}-\mathrm{OH}$. To further confirm this, it is necessary to isolate the fraction with the peak at $m / z 355$ and characterize it with ESR directly. Therefore, the reaction conditions for DCBQ- $t$-BuOOH-BMPO need to be optimized to produce maximal BMPO- ${ }^{-} \mathrm{CBQ}-\mathrm{OH}$. For this purpose, the concentration-dependent effects of the three reactants on the yield of BMPO- ${ }^{\circ} \mathrm{CBQ}-\mathrm{OH}$ were tested (see ESI, $\dagger$ Fig. S1 and S2). Through a series of experiments we found that the optimal reaction conditions are BMPO, $50 \mathrm{mM}$; DCBQ, $1 \mathrm{mM} ; t$-BuOOH $100 \mathrm{mM}$. The effect of solvents was also studied, and acetonitrile was found to markedly enhance the yield of BMPO- ${ }^{-} \mathrm{CBQ}-\mathrm{OH}$ (Fig. S3, ESI + ). It is likely that acetonitrile could slow down the reaction, which is conducive to trapping more - $\mathrm{CBQ}-\mathrm{OH}$ by BMPO.

We have mentioned above that the half life of the nitroxide radical form of $\mathrm{BMPO}^{-} \mathrm{CBQ}-\mathrm{OH}$ is about 5 hours, and it could be readily separated using an analytical column. To get enough pure BMPO- ${ }^{\circ} \mathrm{CBQ}-\mathrm{OH}$ for direct ESR study, semi-preparative HPLC was employed. The fraction with a retention time of $2.62 \mathrm{~min}$ was collected manually with heart cutting (Fig. S4, ESI†).

As expected, a clean six-line ESR signal with equal intensity could be clearly observed with the isolated fraction (Fig. 3), which was found to be corresponding to the unknown peaks in the ESR spectrum of the reaction mixture DCBQ- $t$ - $\mathrm{BuOOH}-$ BMPO. The hyperfine splitting constants of the BMPO- ${ }^{\circ} \mathrm{CBQ}-\mathrm{OH}$ were obtained by simulation: $a^{\mathrm{H}}=25.9 ; a^{\mathrm{N}}=16.04$; and $a^{\mathrm{N}} / a^{\mathrm{H}}=$ 0.62 , which are characteristic of the spin-trapping of a carboncentered quinone ketoxy radical. ${ }^{7,12}$

Compared with our previous research on this topic, ${ }^{7}$ this study made the following new and unexpected discoveries. These include (1) the half-life of the BMPO- ${ }^{\bullet} \mathrm{CBQ}-\mathrm{OH}$ adduct is much longer than that of DMPO (5 h. vs. $15 \mathrm{~min}$ ), which enables further characterization of this adduct; (2) the nitroxide radical form could be distinguished from the nitrone form of BMPO- ${ }^{\circ} \mathrm{CBQ}-\mathrm{OH}$, and both of the two forms could be identified using HPLC/ESI-Q-TOF-MS in this study; in our previous study, only the nitrone form of DMPO- ${ }^{\bullet} \mathrm{CBQ}-\mathrm{OH}$ could be detected and identified; (3) the radical form of $\mathrm{BMPO}-^{-} \mathrm{CBQ}-\mathrm{OH}$ could be directly detected and identified using high resolution FTICR-MS in combination with theoretical calculations, which might be used as a general approach to study other novel radical intermediates; and (4) the radical form of BMPO- $\mathrm{CBQ}-\mathrm{OH}$ could be isolated and purified using semi-preparative HPLC using a neutral mobile phase, and its clean 6-line signal observed directly by ESR without interference of any concurrently formed radicals.

To the best of our knowledge, this study represents the first purification and characterization of the radical form of BMPO- $\mathrm{CBQ}-\mathrm{OH}$ by the complementary application of ESR spin trapping, HPLC/ESI-Q-TOF-MS and FTICR-MS methods, which provided more direct and stronger experimental evidence for the existence of such an unusual quinone ketoxy radical intermediate, and therefore further supporting our previously proposed molecular mechanism for metal-independent decomposition of hydroperoxides by halogenated quinones. ${ }^{5-8}$

This work was supported by NSFC Grants (21237005, 20925724, 21077058 and 21107139) and NIH Grants (ES11497, RR01008 and ES00210) (B.Z.). The authors also acknowledge the technical help provided by Dr H. T. Zhao.

\section{Notes and references}

1 J. L. Bolton, M. A. Trush, T. M. Penning, G. Dryhurst and T. J. Monks, Chem. Res. Toxicol., 2000, 13, 135-160.

2 Y. Song, B. A. Wagner, J. R. Witmer, H. J. Lehmler and G. R. Buettner, Proc. Natl. Acad. Sci. U. S. A., 2009, 106, 9725-9730. 3 B. Meunier, Science, 2002, 296, 270-271.

4 Y. L. Zhao, F. Qin, J. M. Boyd, J. Anichina and X. F. Li, Anal. Chem., 2010, 82, 4599-4605.

5 B. Z. Zhu, H. T. Zhao, B. Kalyanaraman, J. Liu, G. Q. Shan, Y. G. Du and B. Frei, Proc. Natl. Acad. Sci. U. S. A., 2007, 104, 3698-3702.

6 B. Z. Zhu, B. Kalyanaraman and G. B. Jiang, Proc. Natl. Acad. Sci. U. S. A., 2007, 104, 17575-17578.

7 B. Z. Zhu, G. Q. Shan, C. H. Huang, B. Kalyanaraman, L. Mao and Y. G. Du, Proc. Natl. Acad. Sci. U. S. A., 2009, 106, 11466-11471.

8 B. Z. Zhu, L. Mao, C. H. Huang, H. Qin, R. M. Fan, B. Kalyanaraman and J. G. Zhu, Proc. Natl. Acad. Sci. U. S. A., 2012, 109, 16046-16051.

9 H. T. Zhao, J. Joseph, H. Zhang, H. Karoui and B. Kalyanaraman, Free Radicals Biol. Med., 2001, 31, 599-606.

10 B. Halliwell and J. Gutteridge, Free Radicals in Biology and Medicine, Oxford University Press, Oxford, 2007.

11 L. Cui, M. A. Isbell, Y. Chawengsub, J. R. Falck, W. B. Campbell and K. Nithipatikom, J. Am. Soc. Mass Spectrom., 2008, 19, 569-585.

12 A. S. W. Li and C. F. Chignell, J. Biochem. Biophys. Methods, 1991, 22, 83-87. 